\title{
UN ANÁLISIS CENTRO-PERIFERIA PARA LA COMPRENSIÓN DE SISTEMAS ORGANIZACIONALES
}

\author{
Francisco Javier Segura Mojica \\ Instituto Tecnológico de San Luis Potosí, México
}

\begin{abstract}
Resumen.- En este artículo se exploran las relaciones entre el concepto de organización y los conceptos de sistema dinámico y sistema complejo. Posteriormente se emplea el análisis de redes para realizar un análisis del sistema organizacional basado en la existencia de variables nodo que ejercen una fuerte influencia sobre la configuración de su estructura.
\end{abstract}

Palabras clave.- sistema dinámico, análisis de redes, sistema organizacional

A center-periphery analysis for the organizacional systems comprehension.

Abstract.- In this article, the relations between the concept of organization and the concepts of dynamic system and complex system are explored. Later the analysis of networks is used to show a quantitative model based on the existence of variables node that play a strong influence on the configuration of the organizacional structure.

Key words.- dinamyc system, network analisis, organisational system

\section{Introducción}

La dinámica de sistemas aplicada a los estudios organizacionales es un campo de investigación que abre importantes perspectivas para mejorar la comprensión de la evolución de las organizaciones públicas y privadas.

Los modelos cualitativos generados a partir del análisis de sistemas han tenido múltiples aplicaciones en el campo de la organización, entre las que se encuentra el enfoque de aprendizaje organizacional (Senge, 1992); a su vez, los modelos cuantitativos tienen como principal utilidad la formulación de modelos matemáticos útiles para la generación de simuladores organizacionales.

En el presente artículo se exploran las relaciones entre el concepto de organización y los conceptos de sistema dinámico y sistema complejo. Posteriormente se emplea el análisis de redes para proponer un modelo cuantitativo basado en la existencia de variables nodo que ejercen una fuerte influencia sobre la configuración de la estructura organizacional. 


\section{Organizaciones: Sistemas complejos, sistemas dinámicos}

Partiremos del concepto de sistema como un conjunto de elementos interconectados o interrelacionados con un objetivo concreto. Un sistema complejo sería la clase de sistema cuyos vínculos contienen información no visible para el observador y cuyo comportamiento es poco previsible. En un sistema complejo existen propiedades que resultan de las interacciones entre elementos, las cuáles no pueden explicarse a partir de las propiedades de los elementos aislados. Este tipo de atributos se denominan propiedades emergentes.

Esto nos remite al concepto de complejidad, entendida como "el estudio de los principios y las pautas que explican el comportamiento de un fenómeno natural o social desde la perspectiva de la totalidad" (Rivas, 2008).

El estudio de la complejidad tiene la finalidad de entender la estructura del sistema, sus interconexiones y su conducta. Esto ha dado lugar a diferentes aproximaciones en torno a los tipos de complejidad y las propiedades de los sistemas.

Algunas propiedades de los sistemas complejos que podemos observar en las organizaciones y que nos ayudan a entender su naturaleza y comportamiento son la no linealidad (pequeños cambios pueden generar grandes transformaciones); atractores (puntos o estados que atraen a un sistema dinámico hacia si) ${ }^{1}$; autoorganización (capacidad de cambiar su estructura básica en función de su experiencia y el ambiente que le rodea (Heylighen, 1997); redes (agrupación de nodos interconectados entre sí por mecanismos que pueden ser físicos 0 virtuales).

Por otra parte, existen diferentes tipos de complejidad (Aguilera, 2001): estática (relacionada con la función o propósito del sistema); estructural (relacionada con la composición, forma y especialización); o dinámica (se refiere a los aspectos cambiantes en el espacio-tiempo del fenómeno u objeto).

Atendiendo a las definiciones anteriores, podríamos caracterizar a las organizaciones como sistemas complejos con capacidad auto organizativa, que funcionan a través de redes o estructuras cuya no linealidad las hace poco previsibles en el mediano y largo plazo. Su complejidad es de tipo dinámico, ya que es posible identificar diversas situaciones particulares, llamadas estados, que las caracterizan en un punto específico del espacio y del tiempo.

La complejidad dinámica se observa cuando los patrones que permiten determinar el estado siguiente son susceptibles a pequeños cambios en las condiciones

\footnotetext{
${ }^{1}$ De acuerdo con Rivas (2008), los atractores pueden ser de punto fijo o sumidero, cuando atraen el sistema hacia una situación concreta o estado particular, como en el caso de un péndulo; de ciclo límite, cuando el sistema describe un comportamiento periódico, como por ejemplo los ciclos ecológicos; y extraños, cuando no siguen un comportamiento periódico.
} 
iniciales. Este comportamiento puede explicarse a partir de las interacciones no lineales, que causan un comportamiento aperiódico en el comportamiento del sistema complejo (Aguilera, p. 18).

Es en este punto donde conviene mencionar las preguntas que dieron origen a la presente investigación: ¿Se pueden determinar la estructura de relaciones entre las variables de un sistema organizacional?; ¿Se puede medir la intensidad de las relaciones entre las variables que componen un sistema organizacional en un momento determinado?; ¿Diferentes organizaciones tienen estructuras o redes parecidas?;

\section{Organizaciones: ¿Redes de agentes o redes de variables?}

El análisis de redes nos permite abordar el estudio de las organizaciones desde una perspectiva estructural. Una red social se define como un grupo de individuos (nodos) interactúan a través de un conjunto de relaciones interindividuales (Pizarro, 2004).

Según Rivas (2008), las conexiones pueden ser de 5 tipos:

- Burocráticas (órdenes, estandares, políticas, procedimientos).

- Económicas(transacciones comerciales o financieras).

- Operativas (toma de decisiones comunes, recursos compartidos).

- Culturales (valores, normas de conducta).

- Informativas (acceso a fuentes de información) (Rivas, 2008).

Si bien es cierto, que la complejidad de la red está en función de la cantidad de nodos y el número de conexiones posibles (red exponencial), también debemos considerar los mecanismos de conexión; de acuerdo con Kaufman, los organismos se acoplan al entorno con el valor que mas les convenga, seleccionando la estrategia apropiada (Rivas, p. 14). Es decir, emplean mecanismos de conexión basados en conveniencia o economía que les permiten generar órdenes espontáneos, tan simples como sea posible para evitar el caos.

Las redes tienen además una serie de propiedades que permiten comprender su operación:

- Cohesión: Densidad de los nodos que la conforman e intensidad de las relaciones;

- Potencial combinatorio: Cantidad de posibles conexiones que puede establecer un nodo, lo cual responde a la cantidad de nodos y su diversidad.

- Poder de activación: Capacidad de los nodos para conectarse con otros. 
Con base en lo anterior, podríamos caracterizar a las redes como estructuras jerárquicas donde existen nodos que ocupan una posición de centralidad en función de su cohesión con otros nodos, su poder combinatorio y su poder de activación. Estos nodos ejercerían, a su vez, una influencia superior sobre el resto del sistema y sobre sus resultados.

$\mathrm{Si}$, como trataremos de mostrar mas adelante, las organizaciones pueden también ser entendidas como redes donde los nodos, en lugar de individuos, son variables que al igual que aquellos se encuentran interconectadas, entonces el análisis estructural puede ayudarnos a clarificar algunas cuestiones sobre la dinámica de este tipo de sistemas.

Por ejemplo, tomemos el modelo de análisis estructural desarrollado por Borgatti y Everett (1999) basado en la delimitación de un núcleo formado por actores fuertemente correlacionados, así como una periferia en la que predominan actores débilmente vinculados con el resto del sistema (García Muñiz, 2004).

Si para un conjunto de variables organizacionales logramos determinar el número de interconexiones significativas, así como la intensidad de estas, es posible que logremos definir cuáles son las variables clave que se encuentran en el centro de la estructura, y por lo tanto donde se encuentran las zonas de interés, es decir, aquellas donde se observa mayor densidad de interrelaciones, así como la periferia del sistema, es decir, donde las relaciones son mas débiles.

En este caso, nuestro modelo estaría orientado por la variable, no por el actor. La presente investigación pretende esbozar un modelo de red a través del cuál sea posible aproximarnos al funcionamiento de una organización como sistema dinámico mediante la determinación de relaciones estadísticas entre variables información recabada en una muestra de organizaciones.

\section{Desarrollo de la investigación}

Se seleccionaron y operacionalizaron 71 variables de entrada, proceso y salida de un sistema organizacional. Esta selección fue planeada originalmente para explicar el efecto de un sistema de gestión de la calidad (SGC) sobre los resultados de una organización. Sin embargo, al ampliarse la muestra de organizaciones incluyendo empresas que no contaban con un SGC, se consideró la posibilidad de utilizarlas para explicar las interacciones de un sistema organizacional con independencia de que se encontrara en un proceso de gestión de la calidad.

La muestra de organizaciones en la cuál se aplicó el instrumento de recolección de información incluyó 110 empresas manufactureras dedicadas los ramos metalmecánico y automotriz, que fueron seleccionadas aleatoriamente. Con respecto a un universo de 623 establecimientos con 50 o mas trabajadores en el 
estado de San Luis Potosí esta muestra nos permite trabajar con un nivel de confianza de $94 \%$ y un error de $6 \%{ }^{2}$.

Las variables monitoreadas se incluyen en la tabla 1.1 y se encuentran organizadas en 3 categorías, incluyendo variables de entrada, proceso y salida.

Tabla 1.1

1. Tamaño de la empresa

\section{Variables de entrada}

2. Giro de la empresa

3. Antigüedad de la empresa

4. Nivel de implantación del Sistema de Gestión de Calidad (en caso de existir)

5. Conocimiento del entorno

6. Conocimiento de las necesidades de otras empresas cliente

7. Uso de información sobre la imagen de la empresa

8. Posicionamiento con respecto a la competencia

9. Conocimiento del cliente

10. Conocimiento de las necesidades del cliente

11. Comunicación con los clientes

12. Medición de la satisfacción de los clientes

13. Mejora continua a partir de la satisfacción de los clientes

14. Requisitos de selección y contratación

15. Planes de capacitación

16. Sistema de estímulos y recompensas

17. Certificación de capacidades y competencias

18. Gestión de los recursos humanos

19. Gestión de los recursos financieros

20. Gestión de los recursos materiales

21. Misión

\section{Variables de proceso}

22. Visión

23. Política de calidad

2 Tomando como universo una población $\mathrm{N}$ de 623 establecimientos industriales con 50 o mas empleados en San Luis Potosí, se aplicó la siguiente fórmula:

donde

$$
n=\frac{Z^{2} p q N}{N E^{2}+Z^{2} p q}
$$

\footnotetext{
$n$ es el tamaño de la muestra;

$Z$ es el nivel de confianza;

$p$ es la variabilidad positiva;

$q$ es la variabilidad negativa;

$N$ es el tamaño de la población;

$E$ es la precisión o el error.
} 
24. Objetivos

25. Planes, programas y proyectos orientados a la mejora

26. Frecuencia de la capacitación

27. Temas de capacitación

28. Calidad de la capacitación

29. Profesionalización del personal

30. Sistema de capacitación

31. Adopción de nuevas tecnologías

32. Sistemas de información para la producción/prestación de servicios

33. Contenidos de los sistemas de información

34. Situación contractual (estabilidad laboral)

35. Prestaciones económicas

36. Prestaciones sociales

37. Seguridad e higiene en el trabajo

38. Identificación de los productos y/o servicios

39. Interacción de los procesos

40. Planeación de los procesos de producción/prestación del servicio

41. Control de los procesos de producción/prestación del servicio

42. Condiciones para formar equipos de trabajo

43. Actitud de colaboración del personal

44. Comunicación interna

45. Sentido de pertenencia

46. Actitud de servicio

47. Evaluación del personal

48. Evaluación de los servicios administrativos

49. Sistema de evaluación

50. Descentralización en la organización

51. Descentralización en las áreas de trabajo

52. Interés de los líderes por las personas

53. Reconocimiento al personal vinculado al cumplimiento de tareas y objetivos

54. Apertura a la innovación

55. Mecanismos para la mejora continua

56. Indice de satisfacción de clientes

\section{Variables de salida}

57. Satisfacción del personal con respecto a la organización

58. Indicadores de desempeño de los productos/servicios

59. Relación costo-beneficio de los servicios

60. Eficacia de los servicios

61. Trato a los clientes

62. Confiabilidad

63. Capacidad de respuesta

64. Igualdad o equidad

65. Accesibilidad

66. Comunicación

67. Amabilidad 
68. Seguridad

69. Instalaciones

70. Cumplimiento de objetivos

71. Eficacia organizacional

72. Eficiencia organizacional

Cada variable se operacionalizó mediante un proceso de descomposición en factores y subfactores. Esto permitió la construcción de categorías que fueron presentadas al personal de cada empresa en una encuesta con formato de lista de verificación, conforme al modelo de la tabla 1. 2.

Tabla 1.2 Ejemplo de Operacionalización y categorías por variable

\begin{tabular}{|c|c|c|}
\hline Variable & Categorías & Puntos \\
\hline \multirow[t]{4}{*}{$\begin{array}{l}\text { Conocimiento del } \\
\text { entorno }\end{array}$} & $\begin{array}{l}\text { No se cuenta con mecanismos que permitan } \\
\text { conocer las necesidades de la sociedad y el sector } \\
\text { productivo }\end{array}$ & 0 \\
\hline & $\begin{array}{l}\text { Se cuenta con mecanismos para la generación de } \\
\text { la información sobre necesidades pero no se } \\
\text { utilizan para mejorar la operación de la } \\
\text { organización. }\end{array}$ & 1 \\
\hline & $\begin{array}{l}\text { Se cuenta con información sobre las necesidades } \\
\text { y se actualiza periódicamente, pero no se utiliza } \\
\text { para mejorar la operación de la empresa. }\end{array}$ & 2 \\
\hline & $\begin{array}{l}\text { Se cuenta con información sobre las necesidades, } \\
\text { se actualiza periódicamente y se utiliza para } \\
\text { mejorar la operación de la empresa. }\end{array}$ & 3 \\
\hline \multirow{4}{*}{$\begin{array}{l}\text { Conocimiento de } \\
\text { las necesidades } \\
\text { de otras } \\
\text { empresas cliente }\end{array}$} & $\begin{array}{l}\text { No se cuenta con mecanismos para generar de la } \\
\text { información de las necesidades de las empresas } \\
\text { cliente }\end{array}$ & 0 \\
\hline & $\begin{array}{l}\text { Se cuenta con mecanismos para la generación de } \\
\text { la información de las necesidades de las empresas } \\
\text { cliente pero no se utilizan. }\end{array}$ & 1 \\
\hline & $\begin{array}{l}\text { Se cuenta con información sobre las necesidades } \\
\text { de las empresas cliente pero no se utiliza. }\end{array}$ & 2 \\
\hline & $\begin{array}{l}\text { Se cuenta con información sobre las necesidades } \\
\text { de las empresas cliente, se actualiza } \\
\text { periódicamente y se utiliza para mejorar la } \\
\text { operación de la empresa. }\end{array}$ & 3 \\
\hline \multirow{2}{*}{$\begin{array}{l}\text { Uso de } \\
\text { información } \\
\text { sobre la imagen } \\
\text { de la empresa }\end{array}$} & $\begin{array}{l}\text { No se cuenta con mecanismos que permitan } \\
\text { conocer la percepción de la sociedad respecto a } \\
\text { la empresa. }\end{array}$ & 0 \\
\hline & $\begin{array}{l}\text { Se cuenta con los mecanismos para conocer la } \\
\text { percepción de la sociedad respecto a la empresa, } \\
\text { pero no se utilizan. }\end{array}$ & 1 \\
\hline
\end{tabular}




\begin{tabular}{|l|l|l|}
\hline & $\begin{array}{l}\text { Se cuenta con información sobre la percepción de } \\
\text { la sociedad, pero no se utiliza para mejorar a la } \\
\text { empresa. }\end{array}$ & 2 \\
\hline & $\begin{array}{l}\text { Se cuenta con información sobre la percepción de } \\
\text { la sociedad y se utiliza para mejorar a la empresa. }\end{array}$ & 3 \\
\hline $\begin{array}{l}\text { Posicionamiento } \\
\text { de la } \\
\text { competencia } \\
\text { (conocimiento del } \\
\text { mercado) }\end{array}$ & $\begin{array}{l}\text { No se sabe cuáles son las empresas del mismo } \\
\text { giro con las que compite la empresa }\end{array}$ & 0 \\
\cline { 2 - 4 } & $\begin{array}{l}\text { Se sabe cuáles son las empresas del mismo giro } \\
\text { con las que compite la empresa, pero no se } \\
\text { conocen sus servicios ni su calidad }\end{array}$ & 1 \\
\hline & $\begin{array}{l}\text { Se conocen los servicios y la calidad de empresas } \\
\text { con las que compite la empresa, pero no se } \\
\text { comparan con los propios }\end{array}$ & 2 \\
\hline $\begin{array}{l}\text { Se conocen los servicios y la calidad de las } \\
\text { empresas con las que compite la empresa y se } \\
\end{array}$ & $\begin{array}{l}\text { Se conocen los servicios y la calidad de las } \\
\text { empresas con las que compite la empresa, se } \\
\text { comparan con los propios y esto se utiliza para } \\
\text { mejorar. }\end{array}$ & 4 \\
\hline
\end{tabular}

Cada categoría-variable recibió un valor en puntos, basada en la siguiente jerarquización:

0 . El elemento evaluado no existe en la organización.

1. El elemento existe en la organización pero no ha sido medido y/o no se utiliza

2. El elemento ha sido medido y su desempeño es menor a lo esperado

3. El elemento ha sido medido y su desempeño es semejante a lo esperado

4. El elemento ha sido medido y su desempeño es superior a lo esperado y/o se utiliza para la mejora continua

Al ser seleccionada por el personal encuestado fue posible asignar un valor numérico por variable- organización. El criterio para la asignación del valor en puntos fue a mayor intensidad de la variable en la organización, mayor puntaje.

Una vez capturados los valores por variable-organización se generó una matriz de doble entrada con el propósito de mostrar gráficamente los cruces de variables y el correspondiente coeficiente de correlación para cada par ordenado.

Una vez calculados los coeficientes de correlación de cada variable con el resto de las variables del conjunto, se procedió a clasificar los valores de mayor a menor relevancia en cuatro categorías: 
- Relación fuerte:

- Relación moderada:

- Relación débil:

- Relación no significativa:
Coeficiente Pearson superior a 0.6

Coeficiente Pearson superior a 0.5 e inferior a 0.6

Coeficiente Pearson superior a 0.4 e inferior a 0.6

Coeficiente Pearson inferior a 0.4

En los casos donde se identificaron coeficientes Pearson superiores a 0.6 se calculó el coeficiente de correlación ordinal Spearman utilizando el software SPSS 16.0 con el fin de validar los resultados y confirmar la existencia de una relación estadísticamente significativa. Cabe señalar que esto dio origen a una matriz simétrica, ya que por el momento no se determinó la dirección de las conexiones. Sin embargo puede inferirse que estas se dan en un sentido entrada-procesosalidas del sistema.

Con base en esta clasificación se determinó cuáles son las variables con mayor número de relaciones fuertes, moderados y débiles con el resto de los elementos del conjunto.

Tomando como referencia el modelo de Borgatti y Everett, se ordenaron las variables a partir del número y la intensidad de sus correlaciones con otras variables, con el fin de determinar cuáles variables tienden a ubicarse en una zona central del sistema, y por lo tanto tienen mayor influencia sobre sus resultados, y cuáles se encuentran en la periferia del mismo, con poca o nula influencia sobre las salidas del sistema.

Esto permitió construir un modelo gráfico donde cada variable fue identificada con un nodo numerado. En el gráfico 1.1 se muestra el esquema de interacciones; sin embargo, solo se muestran las conexiones consideradas como fuertes, es decir, superiores a 0.6. En el gráfico se puede apreciar las zonas donde existe mayor densidad de relaciones entre variables así como la periferia del sistema, donde existen variables con pocas nulas conexiones con el resto del sistema. El análisis gráfico del sistema se llevo a cabo utilizando el software Visone versión 2.3.5.

\section{Hallazgos}

La investigación que se describe en el presente artículo se encuentra aún en desarrollo, por lo que los hallazgos que se detallan son parciales y tienen relación con el proceso de configuración del modelo organizacional. Nos concentraremos en la descripción de lo que se ha identificado como la parte central del modelo y su periferia.

Entre las variables que muestran un mayor número de conexiones fuertes y moderadas, y que de acuerdo al modelo de Borgatti y Everett representarían la parte central del sistema organizacional, destacan las siguientes: 
Gráfico 1.1 Modelo gráfico de un sistema organizacional

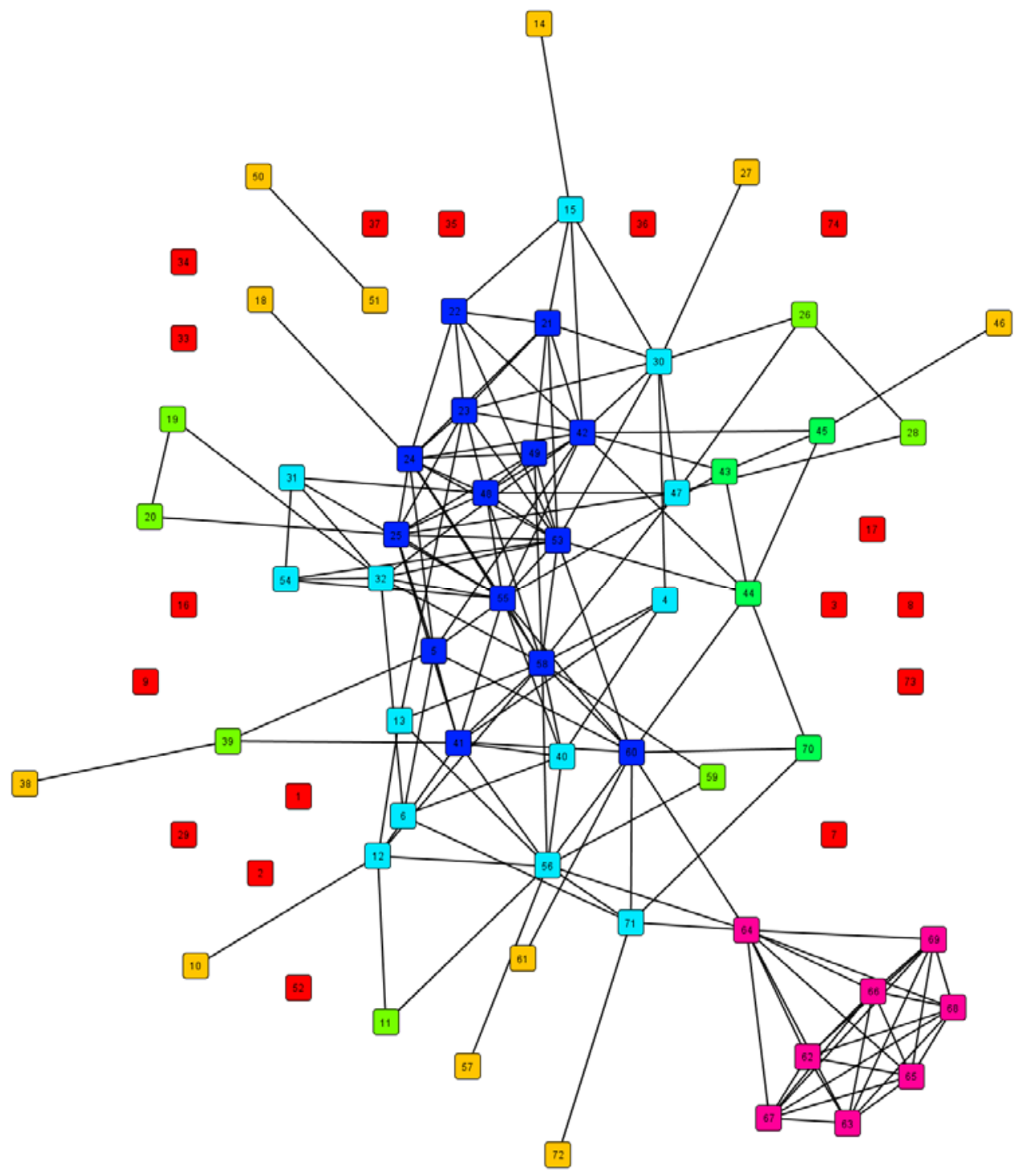

1. La variable reconocimiento al personal vinculado al cumplimiento de tareas y objetivos es la que muestra mayor número de vínculos fuertes, superiores a 0.6 , y moderados, de 0.5 a 0.59 con otras variables. El número de conexiones fuertes es de 16 y moderadas 23.

2. Indicadores de desempeño de los productos/servicios: 15 conexiones fuertes y 22 moderadas

3. Indice de satisfacción de los clientes: 11 fuertes y 22 moderadas

4. Formulación de objetivos: 10 fuertes y 27 moderadas.

5. Mecanismos para la mejora continua: 10 fuertes y 24 moderadas

6. Evaluación de los servicios administrativos: 10 fuertes y 29 moderadas.

7. Planes de mejora: 10 fuertes y 25 moderadas.

8. Política de calidad: 9 fuertes y 28 moderadas 
9. Sistema de capacitación, 9 fuertes y 18 moderadas

10. Misión, 8 fuertes y 13 moderadas.

11. Evaluación del personal: 6 fuertes y 22 moderadas

12. Conocimiento del entorno: 6 fuertes y 20 moderadas

13. Visión: 6 fuertes y 13 moderadas.

14. Medición de la satisfacción de clientes: 6 fuertes y 13 moderadas

Es importante señalar que las variables ubicadas en los lugares 2 y 3 , Indicadores de desempeño de los servicios e Índice de satisfacción de los clientes, están consideradas como variables de salida del sistema, pues se trata de resultados deseables; por lo tanto, el énfasis para la administración debería centrarse en el análisis de las variables de entrada y de proceso que se encuentran conectadas con aquellas.

Sin embargo, el resto de las variables que muestran conexión intensa con el sistema han sido clasificadas como variables de proceso, por lo que un manejo adecuado por la gerencia podría potenciar la capacidad de la organización para generar las respuestas esperadas.

En el extremo opuesto se encuentran las variables con menor número e intensidad de conexiones, como es el caso de Profesionalización del personal (posibilidad de que un empleado realice estudios formales simultáneamente al trabajo), descentralización en la organización, descentralización en el área de trabajo, Interés de los líderes por las personas, situación contractual, prestaciones económicas y prestaciones sociales que muestran un número mínimo de relaciones significativas con otras variables. Este tipo de factores se encontrarían en las zonas periféricas del sistema.

Estos resultados se contrastaron con un análisis de centralidad de cercanía ${ }^{3}$ y de intermediación ${ }^{4}$ desarrollado a través del software Visone. En el gráfico 1.2 se muestran los resultados del análisis de centralidad, donde se pueden identificar claramente 2 bloques de variables fuertemente vinculadas entre si; las variables con valor 5 son las que se encuentran en una posición de mayor centralidad con respecto al sistema. Este grupo incluye, entre otras, las variables reconocimiento al personal vinculado al cumplimiento de tareas y objetivos, conocimiento del entorno, misión, visión y política de calidad.

Existe otro bloque de variables, fuertemente interrelacionadas, pero con solo un enlace hacia el resto del sistema. Estas variables son los atributos de los

\footnotetext{
${ }^{3}$ Esta medición muestra la cercanía o distancia y mide qué tan cerca se encuentra un actor del resto de actores de la red. Cuanto más central sea un actor, mayor será su capacidad de interacción con el resto de actores (Vargas-Quesada 2005).

${ }^{4}$ La interacción entre dos actores no adyacentes puede depender de otros actores de la red, en concreto, de aquellos situados en el path de dichos nodos no adyacentes.
} 
productos o servicios que proporciona la organización y son consideradas como variables de salida del sistema.

\section{Gráfico 1.2 Centralidad de cercanía}

Por otra parte, utilizando el mismo software se analizó la centralidad de

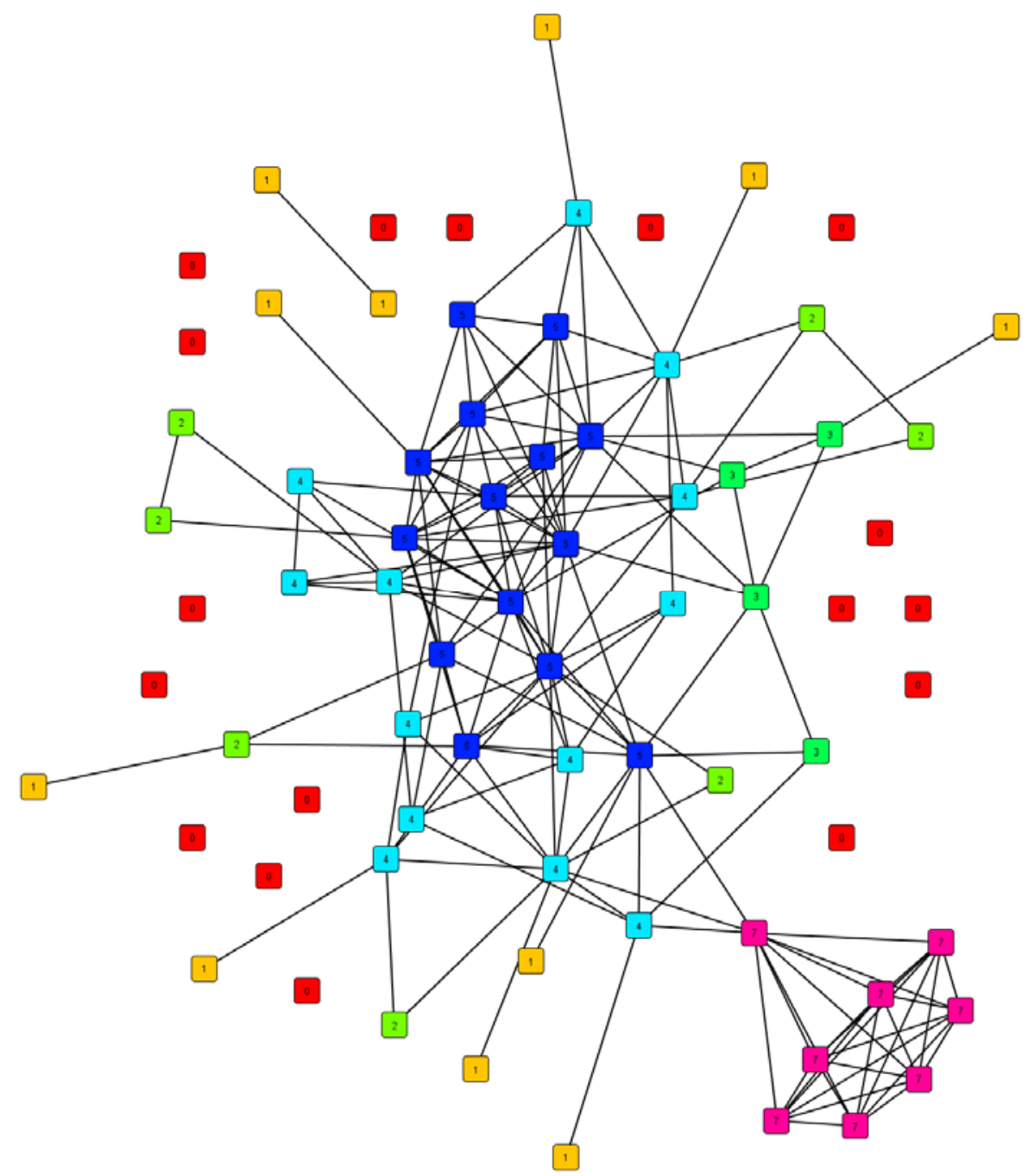

intermediación de las variables, encontrándose que las variables con coeficientes mas altos fueron reconocimiento ligado al desempeño, condiciones para formar equipos de trabajo, indicadores de desempeño de los productos/servicios y eficacia de los productos/ servicios. Este tipo de actores o intermediarios de paths entre actores, son los encargados de controlar las interacciones en la red ya que "la centralidad por intermediación o betweenness mide el grado en que un actor concreto -intermediario- forma parte del path más corto o distancia geodésica entre otros actores" (Vargas-Quesada 2005). 


\section{Gráfico 1.3 Centralidad de intermediación}

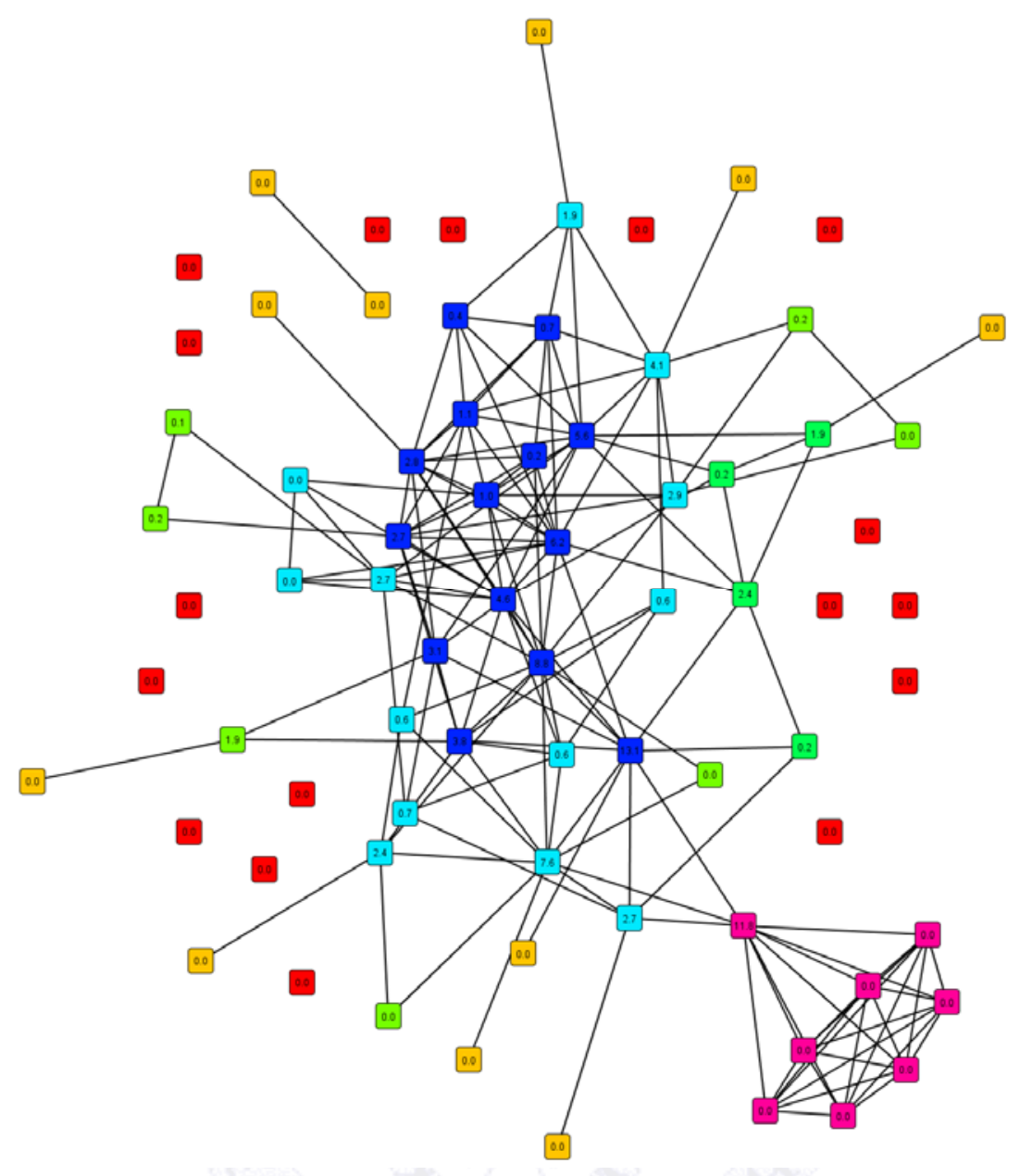

\section{Conclusiones}

Los resultados presentados en el presente reporte son parciales, ya que la finalidad de la investigación es lograr la formulación de un modelo que permita lograr una aproximación consistente a la estructura de los sistemas organizacionales.

Sin embargo, de las observaciones realizadas se pueden desprender las siguientes consideraciones:

1. Existe un núcleo organizacional constituido por las variables que muestran mayor número e intensidad de conexiones significativas.

2. En este núcleo destacan los aspectos relacionados con el reconocimiento al personal en función del cumplimiento de tareas, la formulación de misión, visión y objetivos, los mecanismos de mejora continua y la 
evaluación del personal y de los servicios. En otras palabras, el énfasis de este modelo organizacional se encuentra en el enfoque hacia el cumplimiento de tareas y objetivos.

3. En contraste, el énfasis hacia el factor humano, que se muestra en variables como interés por las personas, descentralización o impulso a la profesionalización del personal, se encuentra en la periferia del sistema, con pocas conexiones significativas hacia los resultados o salidas de la organización.

4. Lo anterior nos muestra que en la práctica, la mayoría de las organizaciones analizadas gestionan su capital humano en función de los requerimientos de la tarea y en esa medida se proporcionan reconocimiento y capacitación. Sin embargo otros aspectos ligados al desarrollo humano como sería el estilo de liderazgo aplicado por la gerencia o las facilidades para realizar estudios profesionales, no estarían conectados con los resultados organizacionales.

5. La formulación de misión, visión, política de calidad, pero sobre todo objetivos, evaluación y reconocimiento en función de resultados, representan importantes herramientas para el logro de los resultados organizacionales esperados. Estos elementos representan la columna vertebral de la organización como sistema y probablemente sean claves para explicar su dinámica y prever sus resultados.

\section{Bibliografía}

(2001) Aguilera, Antonio; Lopez, Adolfo. "Modelado multiagente de sistemas socioeconómicos: una introducción al uso de la inteligencia artificial en la investigación social". El Colegio de San Luis A.C . San Luis Potosí, México.

(1999) Borgatti, Stephen P., Everett, Martin G. "Models of core/periphery structures". Social Networks, No. 21, pp. 375-395.

(2005). Brandes, Ulrick; Kenis, Patrick. La explicación a través de la visualización de redes. REDES- Revista hispana para el análisis de redes sociales, Vol.9,\#6.

(2004) García Muñiz, Ana Salomé, et. al. "Estimación y evaluación de modelos estructurales centro periferia". REDES Revista hispana para el análisis de redes sociales" Vol. 6. No. 5.

(2003) Moriello, Sergio. Sistemas complejos, caos y vida artificial. Revista Electrónica Red Científica. Madrid: Enk3 comunicación.

(2004) Pizarro, Narciso. "Un nuevo enfoque sobre la equivalencia estructural: lugares y redes de lugares como herramientas para la teoría sociológica". REDES Revista hispana para el análisis de redes sociales Vol. 5 No. 2. 
(2008) Rivas, Luis Arturo. "Los 10 conceptos básicos para entender la teoría de la complejidad y sus implicaciones en la gestión de las organizaciones".Memoria del XII Congreso Internacional en Ciencias Administrativas-ACACIA.

(1992) Senge, Peter. "La Quinta disciplina: el arte y práctica de la organización inteligente". Buenos Aires: Granica.

(1994) Sterman, John. "Learning in and about complex systems". System Dynamics Review 10(2-3): 291-330.

(2002) Snijders, Tom. "Macro-micro-macro y modelos estadísticos para redes". REDES Revista hispana para el análisis de redes sociales Vol. 3 No. 3.

(2005) Vargas-Quesada, Benjamín. Visualización y Análisis de Grandes Dominios Científicos Mediante Redes PathFinder. Granada: Universidad, Tesis Doctoral,. 